\title{
Design and Validation of Thin-Walled Composite Deployable Booms with Tape-Spring Hinges
}

\author{
H.M.Y.C. Mallikarachchi* \\ University of Cambridge, Cambridge CB2 1PZ, U.K. \\ S. Pellegrino ${ }^{\dagger}$ \\ California Institute of Technology, Pasadena, CA 91125
}

\begin{abstract}
This paper presents a $1 \mathrm{~m}$ long self-deployable boom that could be folded around a spacecraft. Previously developed simulation techniques are used to analyze this two-hinge boom, made from two-ply plain weave carbon fiber laminate. A stress-resultant based failure criterion is used to study safety of the structure during both stowage and dynamic deployment. A safe design that latches without any overshoot is selected and validated by a dynamic deployment experiment.
\end{abstract}

\section{Introduction}

Composite booms with tape-spring hinges offer several advantages, such as lightness, efficient packaging, low cost and deployment behavior insensitive to friction. They were recently used for the deployable antennas on MARSIS ${ }^{1}$ and a number of applications for small satellites have been proposed. Examples of recent technology developments for future missions are Ruag's self-motorized deployment mechanism, ${ }^{2}$ DLR's lightweight deployable booms, ${ }^{3,4}$ the Phased-Array-Fed Reflector for the Innovative Space-Based Radar Antenna Technology (ISAT) program. ${ }^{5}$

The deployment scheme typically envisages the release of all constraints on the folded boom, to allow the structure to dynamically deploy and self latch. However this behavior needs to be fully understood and optimized as severe dynamic effects at the end of deployment could damage the structure and yet a slow, highly damped deployment may end without ever achieving the fully deployed configuration. Achieving a balance between these effects is challenging, as demonstrated by the large amount of testing and simulation that was required to achieve the successful deployment of the MARSIS booms. ${ }^{6}$

Here we consider a simple boom construction based on a thin-walled tube made of two plies of plain-weave carbon fiber in an epoxy matrix. Certain regions of the tube are weakened by cutting away some of the composite material to form tape-spring hinges at which the tube can be folded without causing any damage. We use a set of analysis tools that have been recently developed ${ }^{7-10}$ to arrive at a specific design for a $1 \mathrm{~m}$ long tubular boom with a diameter of $38 \mathrm{~mm}$ and a thickness of $0.22 \mathrm{~mm}$ (these properties were chosen for the sake of definiteness) that can be wrapped around a spacecraft and is able to dynamically self-deploy without damage. This design is then verified by constructing and testing this boom.

A brief review of the simulation techniques, including modeling of a thin laminate by homogenization of a periodic unit cell and finite-element simulation of quasi-static folding and dynamic deployment with Abaqus/Explicit, are provided in Section II. The numerical simulations are integrated with a material failure criterion, presented in Section III, based on six stress-resultants for the specific two-ply CFRP laminate that is of interest. The combination of these tools allows us to analyse the detailed effects of hinge design changes and find a design that can be safely folded and deployed, in Section IV. Section V presents an experimental verification of the chosen design by carrying out an actual deployment experiment. Section VI concludes the paper.

${ }^{*}$ Research Student, Department of Engineering, Trumpington Street, Cambridge CB2 1PZ, U.K. Currently at California Institute of Technology

${ }^{\dagger}$ Joyce and Kent Kresa Professor of Aeronautics and Professor of Civil Engineering, Graduate Aerospace Laboratories, 1200 E. California Blvd. MC 301-46. AIAA Fellow. sergiop@caltech.edu 


\section{Review of Simulation Techniques}

Recently, we have developed several analysis tools for self-deployable composite structures and here we give a brief review of our earlier work.

\section{A. Laminate Model}

Thin laminates made of woven tows of carbon fiber are not accurately modeled by classical lamination theory. ${ }^{11}$ A more accurate model is a linear-elastic thin Kirchhoff plate whose properties are defined by a homogenization technique, based on the geometric properties of the laminate measured from micrographs and tow properties calculated from appropriate rules of mixtures.

The repeating unit cell is modeled with triangular prism elements and periodic boundary conditions are applied, Figure 1. After carrying out six separate finite element analyses (we use the package ABAQUS/Standard), each corresponding to a unit amplitude of the six deformation variables (three mid-plane strains and three curvatures) using virtual work, the material stiffnesses can be computed in the form of an ABD matrix, which is then provided to ABAQUS/Explicit, see the next section, to define the shell stiffness. A more detailed description of the laminate model is presented in Reference. ${ }^{10}$

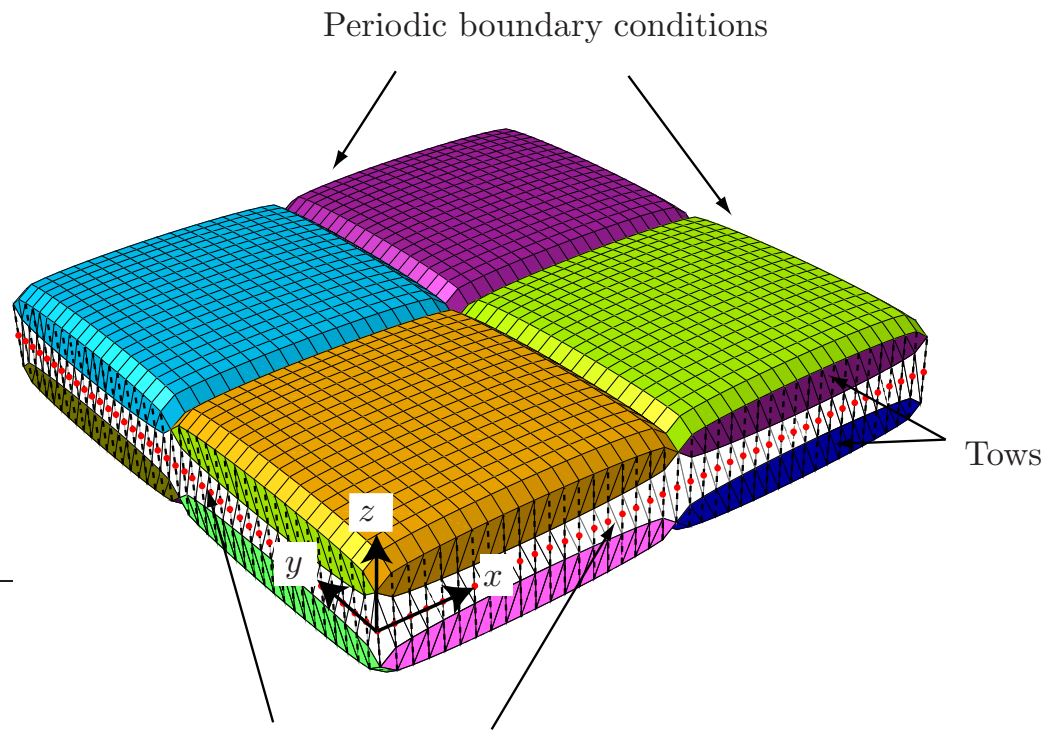

Periodic boundary conditions

Figure 1: Finite element model for unit cell of two-ply laminate (with 3X magnification in $z$-direction).

\section{B. Simulation Techniques}

Folding and deployment of thin-walled composite structures involve significant geometric changes that are associated with instabilities, dynamic snaps and extensive contact/sliding between different parts of the structures. The ABAQUS/Explicit ${ }^{12}$ commercial finite element package has been used to capture all of these features. The use of an explicit solver removes the convergence difficulties caused by singularities in the stiffness matrix as it advances the kinematic state of each degree of freedom by direct integration of its equations of motion.

However careful is needed when using this approach. Explicit time integration is only conditionally stable and hence one needs to maintain the minimum time increment:

$$
\Delta t=\alpha\left(\sqrt{1+\xi^{2}}-\xi\right) \frac{l_{\min }}{c_{d}}
$$

with the time scaling factor $\alpha \leqslant 1$ and $\xi=$ fraction of critical damping in highest frequency mode, $l_{\min }=$ shortest length of finite element and $c_{d}=$ wave speed through material. But satisfying this condition does 
not guarantee a solution free of instabilities. The best way to ensure this is to check that energy conservation is satisfied. ${ }^{13}$ In other words, the energy balance term, defined as the summation of the internal energy, kinetic energy and viscous dissipation minus the work done by the external forces, has to be constant when energy is not supplied to the system.

The composite boom is modeled in ABAQUS/Explicit using four-node fully integrated shell elements and the ABD stiffness matrix (obtained in the previous section) is incorporated by means of the ${ }^{*}$ Shell General Section definition. The ${ }^{*}$ General Contact feature automatically defines all potential contact surfaces.

Quasi-static folding simulations are carried out by prescribing any changes in boundary conditions as smooth fifth-order polynomial functions of time (Smooth step) to minimize accelerations everywhere in the structure. The validity of a quasi-static simulation is assumed if the kinetic energy to internal energy ratio is less than $1 \%$. The main simulation parameters and the values to be used in these type of analysis are presented in Reference. ${ }^{10}$ In the present case the detailed steps of folding are not important provided that the kinetic energy in the final folded configuration is negligible. Therefore the structure is folded as fast as possible to minimize computational cost but with a smooth step definition to achieve low accelerations at the end of the step. Then an additional $0.2 \mathrm{~s}$ simulation is carried out by applying higher viscous pressure on all external surfaces of the structure to quickly damp out any remaining kinetic energy.

Once the static folded configuration is obtained folding restraints are released to allow dynamic deployment to occur and a linear bulk viscous pressure is applied to damp high frequency oscillations. ${ }^{8}$

One of the outcomes of these simulations are the mid-surface stress resultants, i.e. the mid-plane forces and moments per unit length in the homogenized shell structure, as well as the mid-plane strains and curvatures, everywhere in the structure and for all configurations. Critical locations can be identified by analyzing these sets of stress resultants with the failure criterion of Section III.

\section{Failure Criterion}

In previous publications ${ }^{7-10}$ we have used the homogenization analysis in reverse to determine the maximum strains in the fibers and the matrix from the mid-plane strains and curvatures. We have found that this approach is of limited accuracy, particularly when there is a combination of stress resultants acting on a thin laminate. Also, it has been observed that thin composites can be subjected to higher surface strains than ultimate fiber strains ${ }^{14,15}$ without apparent failure. For these reasons, instead of these micro-mechanics based approaches, we will use a six dimensional failure locus defined in terms of force and moment resultants.

A six-dimensional failure locus can be defined from five uniaxial strength values that can be obtained from tensile, compressive, shear, bending and twisting failure tests. For a symmetric two-ply plain weave laminate made from Toray T300-1k fibers and Hexply 913 epoxy resin the uniaxial strengths are given in Table 1.

\begin{tabular}{|l|c|}
\hline Strength & Value \\
\hline Tensile, $F_{1 t}=F_{2 t}(\mathrm{~N} / \mathrm{mm})$ & 139.47 \\
Compressive, $F_{1 c}=F_{2 c}(\mathrm{~N} / \mathrm{mm})$ & 63.42 \\
Shear, $F_{3}(\mathrm{~N} / \mathrm{mm})$ & 17.73 \\
Bending, $F_{4}=F_{5}(\mathrm{Nmm} / \mathrm{mm})$ & 3.04 \\
Twisting, $F_{6}(\mathrm{Nmm} / \mathrm{mm})$ & 0.92 \\
\hline
\end{tabular}

Table 1: Uniaxial strength properties of two-ply T300-1k/Hexply 913 laminate.

The failure locus is defined by the three inequalities:

$$
\begin{array}{r}
J_{1}\left(N_{x}+N_{y}\right)+K_{11}\left(N_{x}^{2}+N_{y}^{2}\right)+K_{12} N_{x} N_{y}+K_{33} N_{x y}^{2}<1 \\
K_{44} \times \max \left(M_{x}^{2}, M_{y}^{2}\right)+K_{66} M_{x y}^{2}<1 \\
\max \left(\frac{N_{x}}{F_{x}}, \frac{N_{y}}{F_{y}}\right)+\frac{\max \left(\left|M_{x}\right|,\left|M_{y}\right|\right)}{F_{4}}<1
\end{array}
$$


where the subscripts $x$ and $y$ refer to the tow directions and

$$
\begin{aligned}
J_{1} & =\frac{1}{F_{1 t}}-\frac{1}{F_{1 c}} \\
K_{11} & =\frac{1}{F_{1 t} F_{1 c}} \\
K_{33} & =\frac{1}{F_{3}^{2}} \\
K_{44} & =\frac{1}{F_{4}^{2}} \\
K_{66} & =\frac{1}{F_{6}^{2}} \\
K_{12} & =-\frac{K_{11}}{2}
\end{aligned}
$$

and $F_{x}, F_{y}$ are the axial failure strengths, given by

$$
\begin{aligned}
& F_{x}=\frac{-\left(J_{1}+K_{12} N_{y}\right) \pm \sqrt{\left(J_{1}+K_{12} N_{y}\right)^{2}-4 K_{11}\left(J_{1} N_{y}+K_{11} N_{y}^{2}+K_{33} N_{x y}^{2}-1\right)}}{2 K_{11}} \\
& F_{y}=\frac{-\left(J_{1}+K_{12} N_{x}\right) \pm \sqrt{\left(J_{1}+K_{12} N_{x}\right)^{2}-4 K_{11}\left(J_{1} N_{x}+K_{11} N_{x}^{2}+K_{33} N_{x y}^{2}-1\right)}}{2 K_{11}}
\end{aligned}
$$

Note that Equation 4(a) is obtained by setting $N_{x}=F_{x}$ and replacing $<$ with $=$ in Equation 2a, and then solving the quadratic equation for $F_{x}$. Similarly, Equation $4(\mathrm{~b})$ is obtained by setting $N_{y}=F_{y}$, etc. and then solving for $F_{y}$.

Figures 2-4 show a set of interaction diagrams between pairs of stress resultants. The interaction between mid-plane forces follows the Tsai-Wu failure criterion, ${ }^{16}$ Figure 2 . Bending moments along tow directions at $90^{\circ}$ are independent from one another but each interacts quadratically with the twisting moment, Figure 3. Lastly, the axial forces and bending moments interact linearly, see Figure 4.

In addition to the failure locus determined from Equations 2, 3 and 4, each diagram contains several experimentally determined failure points that were determined from combined loading tests. A detailed description of this failure locus will be presented elsewhere. ${ }^{17}$

It should be noted that the ABD stiffness matrix obtained from the laminate model is for the initial geometry of the tows, but experiments have shown that there is a gradual reduction in bending stiffness and close to failure this is $40 \%$ lower than in the unloaded conditions. This reduction factor was evaluated by comparing the moment at failure, obtained experimentally, to the moment calculated by multiplying the corresponding curvature at failure with the initial ABD stiffness matrix. Because all simulations carried out in this paper use the initial ABD matrix, they will overestimate the bending and twisting moments near failure and to correct for this in an approximate way both bending and twisting strengths were multiplied by a factor of 1.67 when analyzing the stress resultants from the simulations. The increased bending and twisting strengths are $F_{4}^{\prime}=F_{5}^{\prime}=5.07 \mathrm{Nmm} / \mathrm{mm}$ and $F_{6}^{\prime}=1.53 \mathrm{Nmm} / \mathrm{mm}$.

The tensile and compressive responses were almost linear up to failure and hence no modifications of the corresponding strengths were made.

\section{Boom Design}

We have considered a deployment scheme that requires a $1 \mathrm{~m}$ long monolithic boom with two tape-spring hinges, to be folded around a small spacecraft, as shown Figure 5. The boom is rigidly connected to the spacecraft and expected to self-deploy upon release. It is also required to fully latch straight away, without overshooting, as soon as it becomes fully deployed to avoid any interference with the spacecraft itself or other equipment attached to it. The spacecraft had cross-sectional dimensions of $400 \mathrm{~mm}$ by $360 \mathrm{~mm}$ and the centers of the two hinges were placed at distances of $200 \mathrm{~mm}$ and $600 \mathrm{~mm}$ from the root end. The distance between the root and the first hinge was chosen such as to allow the boom cross-section to deform near the hinge. 


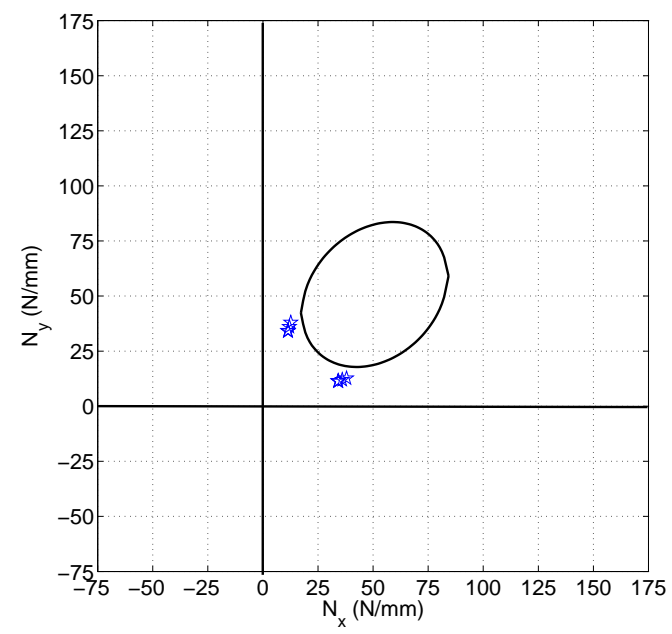

(a) $N_{x}$ and $N_{y}$ when $N_{x y}=20.38 \mathrm{~N} / \mathrm{mm}$

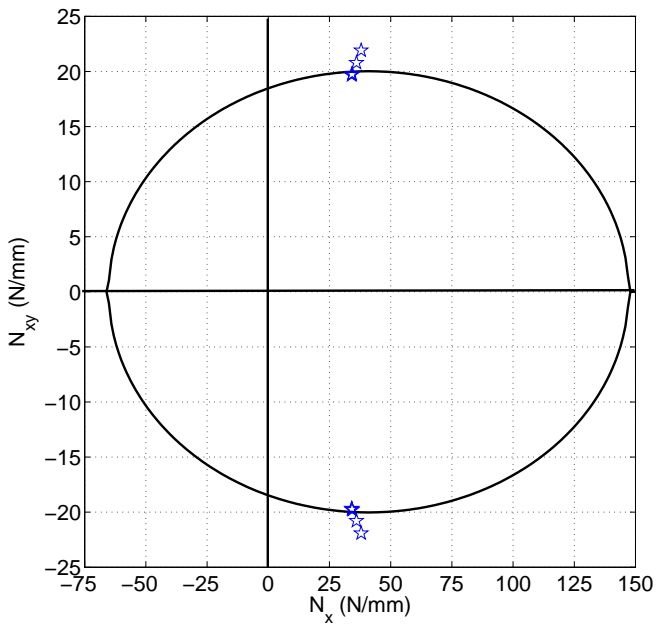

(b) $N_{x}$ and $N_{x y}$ when $N_{y}=11.77 \mathrm{~N} / \mathrm{mm}$

Figure 2: Interactions between force resultants.

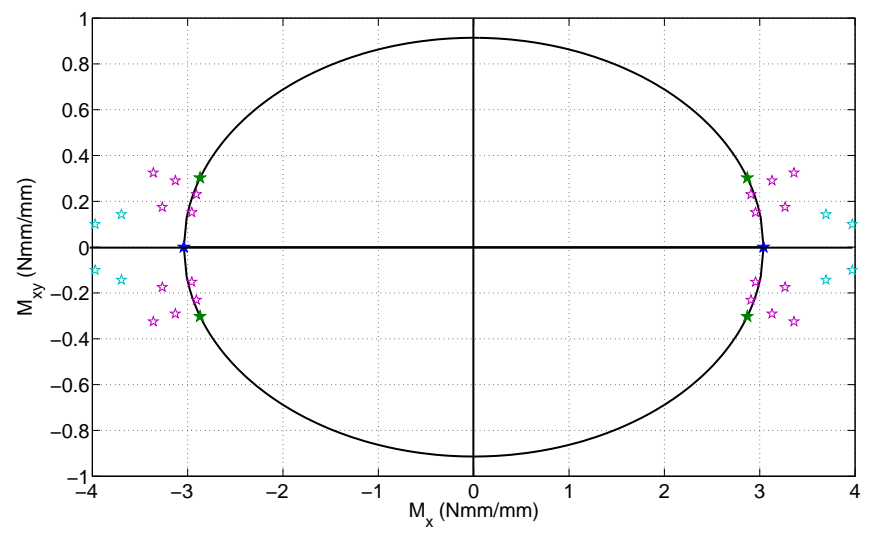

Figure 3: Interaction between bending moment and twisting moment. 


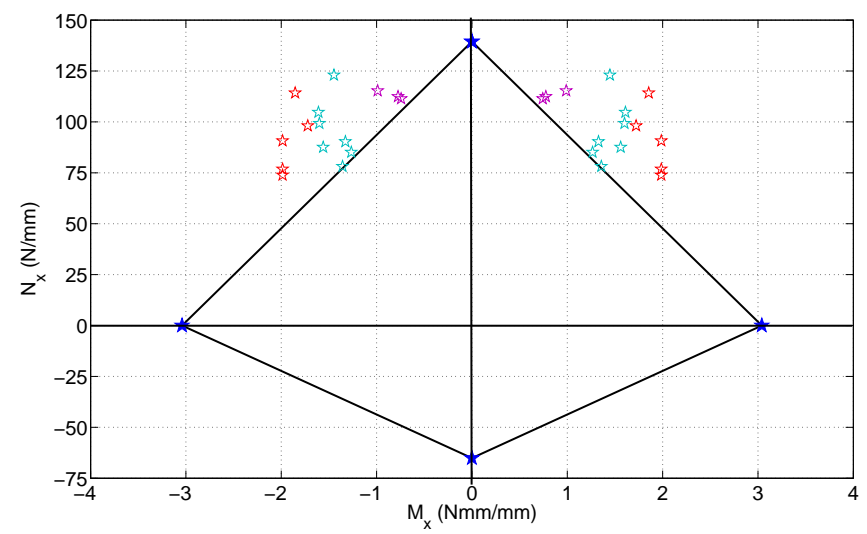

Figure 4: Interaction between axial forces and bending moments.

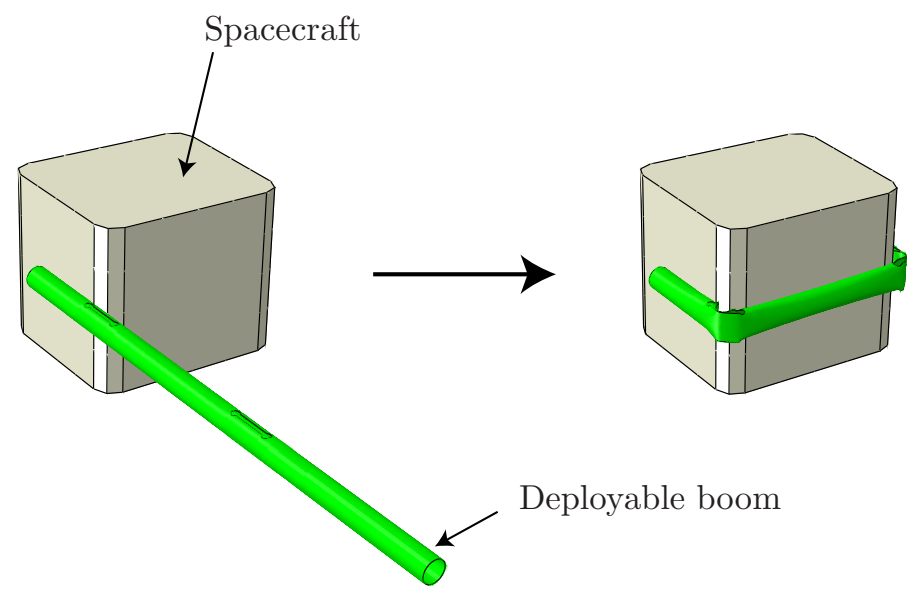

Figure 5: Deployable boom mounted on small spacecraft. 
It would not be practical to analyze in full the folding and deployment behavior of many booms with different hinge designs. Instead, we started from the results presented in Reference ${ }^{9}$ which had considered a general tape-spring hinge geometry defined by three parameters, slot length, $L$, slot width, $S W$ and end circle diameter, $D$, Figure 6 . This study carried out a sensitivity study of the hinge to these parameters and concluded that a good hinge design for folding angles up to $180^{\circ}$ has $L=90 \mathrm{~mm}, S W=8 \mathrm{~mm}$ and $D=$ $15 \mathrm{~mm}$. However, it should be noted that in this study the hinge cross-sections were left unconstrained and so were allowed to ovalize.

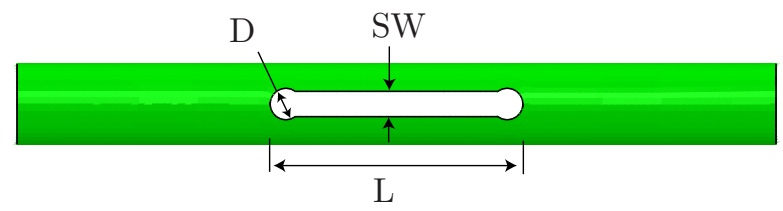

Figure 6: General hinge geometry.

Based on these previous results ${ }^{9}$ we considered three possible hinge designs, with the slot parameters defined in Table 2. In the next sections we will present our detailed analyses of a boom based on the first hinge design, in Sections A-C, and then describe compare key results for all three designs in Section D to finally choose a design to be taken forward.

\begin{tabular}{|c|c|c|c|}
\hline \multirow{2}{*}{ Design } & \multicolumn{3}{|c|}{ Slot parameters } \\
\cline { 2 - 4 } & $L(\mathrm{~mm})$ & $S W(\mathrm{~mm})$ & $D(\mathrm{~mm})$ \\
\hline I & 90 & 10 & 15 \\
II & 85 & 10 & 15 \\
III & 90 & 8 & 15 \\
\hline
\end{tabular}

Table 2: Slot parameters for three hinge designs.

\section{A. Finite Element Model}

The complete model of the boom consisted of 17644 nodes and 17400 four node fully integrated shell elements, S4. The finite element mesh was finer over the hinge regions, to capture the details of the complex deformation that occurs in these regions. The spacecraft was modeled with 112 reduced integration eight node brick elements, C3D8R, which were defined to form a single rigid body connected to a reference node. Fixed boundary conditions were specified at the root of the boom and also at the reference node of the rigid block representing the spacecraft. Contact between the boom and the spacecraft and self contact of different parts of the boom was defined with friction less ${ }^{*}$ General Contact definition . Figure 7 shows the complete model.

The first part of the simulation generated the folded configuration of the boom. The detailed sequence of steps to achieve this configuration is less important than the final folded configuration itself, provided that the kinetic energy in the folded configuration is sufficiently small and the energy balance term remains constant throughout. Because the hinges have much higher bending stiffness in the fully deployed configuration, the folding process was started by pinching each hinge in the middle. This was done by applying equal and opposite forces $P_{1}$, at the three locations, one at the center of each hinge and the other two on either side at a distance of $5 \mathrm{~mm}$. Then two additional forces, $P_{2}$, were applied normal to the boom in the negative $Y$-direction at of $400 \mathrm{~mm}$ and $800 \mathrm{~mm}$ from the root of the boom. All of these forces remain normal to the boom throughout the folding simulation, and eventually wrap the boom around the spacecraft. Figure 8 shows different stages of folding sequence. A small viscous pressure with viscous pressure coefficient equal to $2 \times 10^{-4}$ the density times the dilatational wave speed through the material, $\rho c_{d}$ was applied over the external surface of the boom throughout the entire folding simulation to numerically damp out any stress waves. $^{12}$

Deployment was initiated by the sudden removal of the forces $P_{1}$ and $P_{2}$. At this stage linear bulk viscosity factor was set to 0.1 and viscous pressure loading with coefficient equal to $2 \times 10^{-4} \times \rho c_{d}$ was applied only 


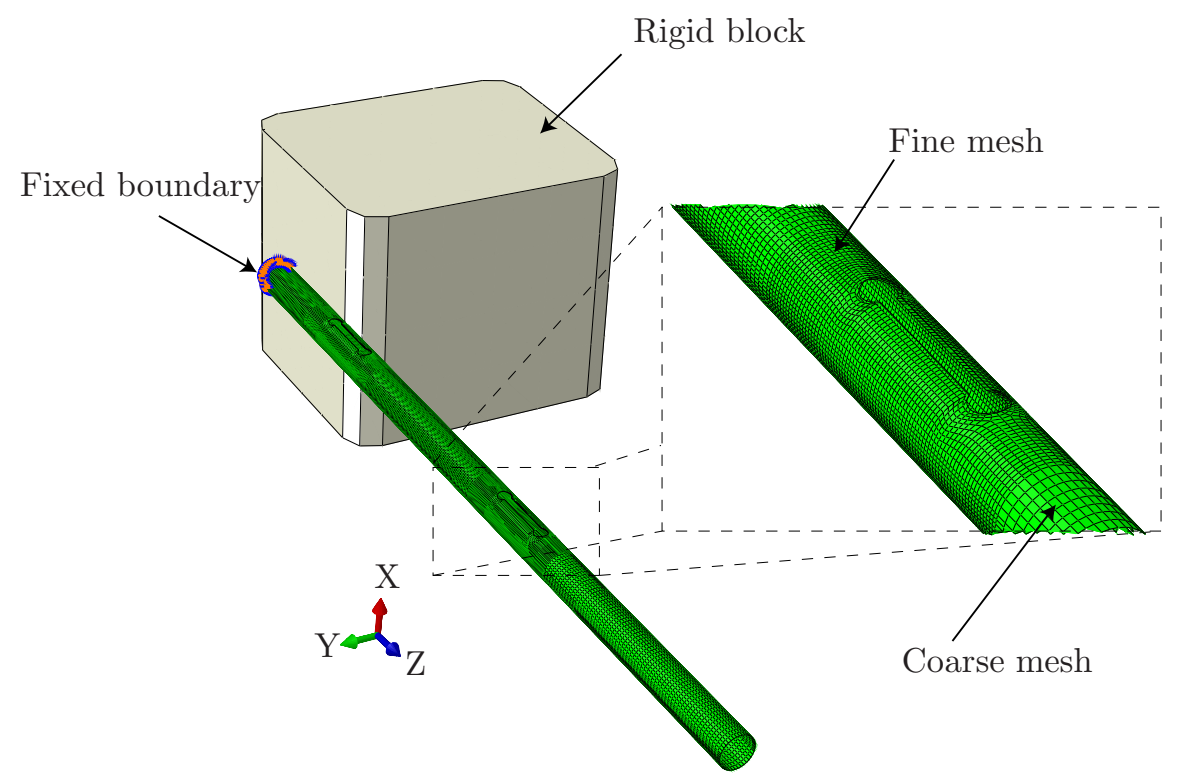

Figure 7: Finite element model of spacecraft and deployable boom.

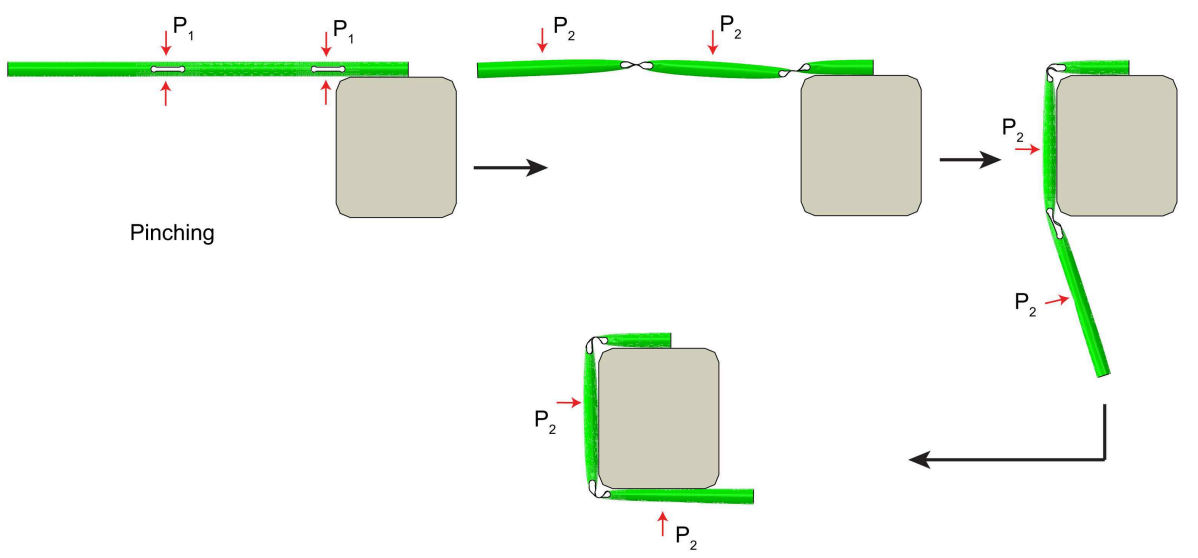

Figure 8: Folding sequence. 
to the hinge regions until each hinge became fully latched, to suppress artificial high frequency oscillations. One needs to be careful in using viscous pressure as it can overdamp the structural response. A detailed description of how to set the simulation parameters and choose the required mesh density is provided in our previous papers. ${ }^{8-10}$

Figure 9 shows the energy variation of the complete simulation. Note that the kinetic energy is almost zero at the beginning of the deployment stage and the energy balance term remains almost constant throughout the entire simulation.

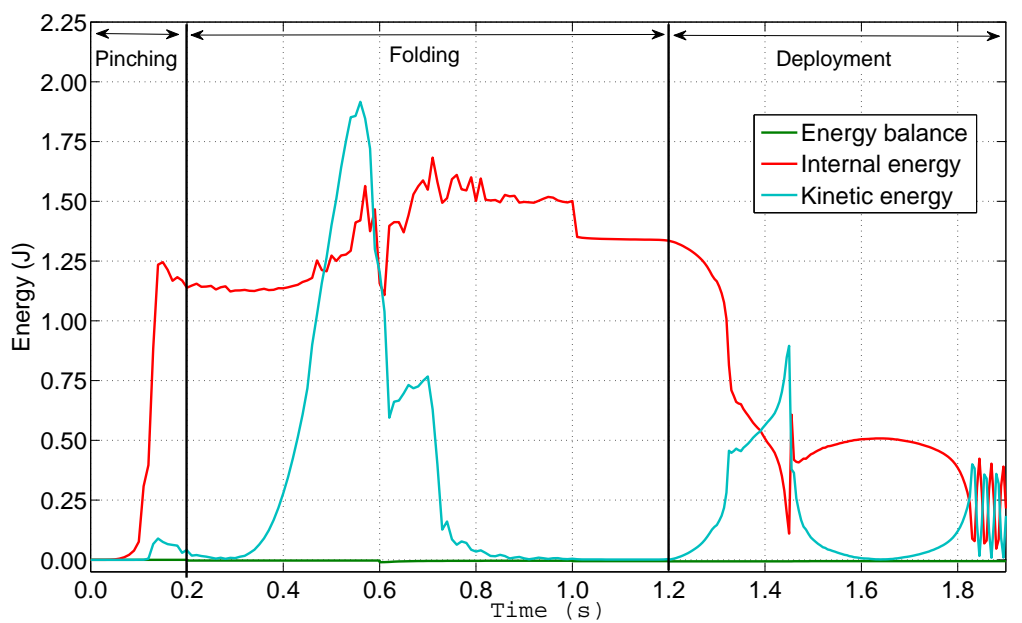

Figure 9: Energy variation for full folding and deployment simulation of boom with hinges based on design I.

\section{B. Dynamic Deployment Behavior}

Figure 10 shows snapshots from the dynamic deployment of the boom with hinges based on design I. Note that the hinge closer to the free end latches first while the rest of the boom remains almost stationary; then the root hinge starts deploying. Figure 11 shows angle-time responses for the two hinges. Also note that both hinge angles are defined with respect to the axis of the boom at the root and hence the two angles approximately coincide after the second hinge has deployed.

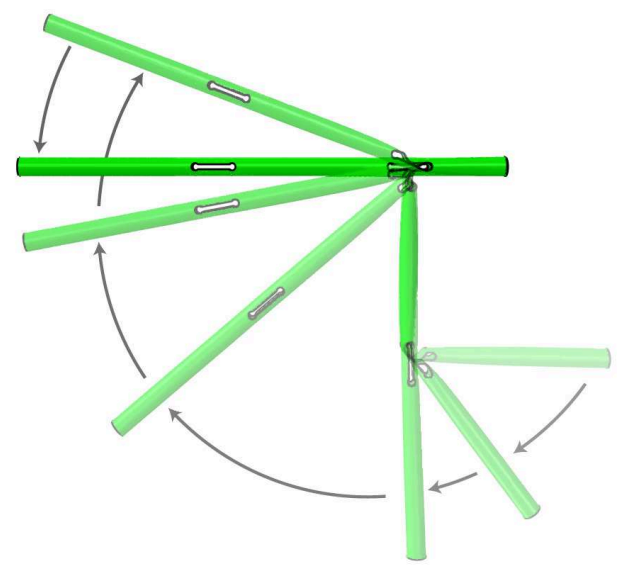

Figure 10: Snapshots during deployment (design I). 


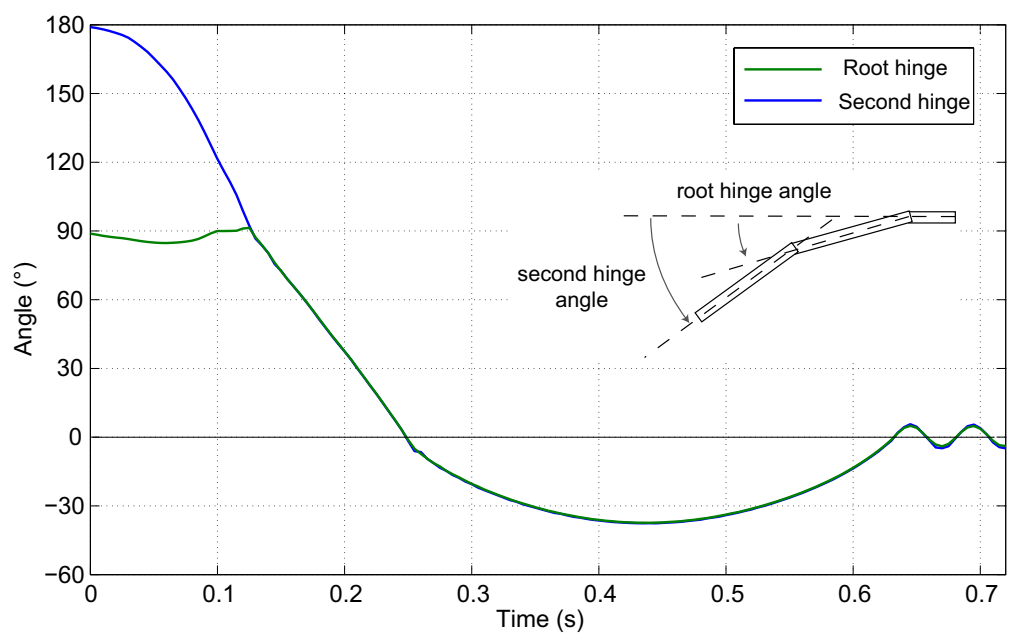

Figure 11: Angle-time response (design I).

In this particular hinge design the root hinge is unable of resisting the angular momentum of the boom at the point of latching, hence the boom overshoots the fully deployed configuration. This behavior should be avoided because the boom could become damaged, or could interfere with the spacecraft.

\section{Failure Analysis}

A failure analysis was performed by applying the failure criterion presented in Section III to the stressresultants obtained from the finite element simulations. Three failure indices, FI-1, FI-2 and FI-3, were defined by evaluating the left hand sides of Equations 2a, 2b and 2c, respectively, and their values were calculated at every integration point and averaged over each element. These three indices are useful to understand the dominant loading condition at each point and, to satisfy the failure criterion, all three values should be less than 1 .

A complete failure analysis of a particular boom design consists of two parts. Firstly, the boom should be safely foldable around the spacecraft and, secondly, it needs to withstand the dynamic loads during deployment.

Figure 12 shows contours of the three failure indices for design I in the fully folded configuration of the boom. The top set of pictures represent the root hinge and the bottom set represent the second hinge. Note that the root hinge is subjected to higher loads than the other hinge. This is not surprising, because the fixed connection at the root of the boom restricts deformation of the boom cross-section whereas the second hinge is less constrained. Also note that the largest failure index is FI-3, which indicates that this boom design is governed by the interaction between axial and bending loads.

The failure analysis during deployment has to calculate the failure indices throughout the entire deployment simulation and then consider their maximum values. Hence, the stress-resultants were recorded at intervals of $0.005 \mathrm{~s}$ for the entire deployment simulation and the three failure indices were calculated. The present hinge design reaches its most critical conditions during the snapping of the second hinge, with FI-3 going from 1.13 to 1.15 during this snap, see Figure 13. Once the second hinge has latched FI-3 begins to decrease, see the image on the right in Figure 13.

\section{Selection of Tape-Spring Hinge Design}

Table 3 lists the maximum failure indices, obtained for each of the three designs, both in the fully folded configuration and during deployment; the critical failure index is always FI-3, hence the dominant loading condition is the interaction between axial and bending loads. Note that the values during deployment are largest for all three designs, the corresponding locations of these maximum failure index are shown in Figure 14. 


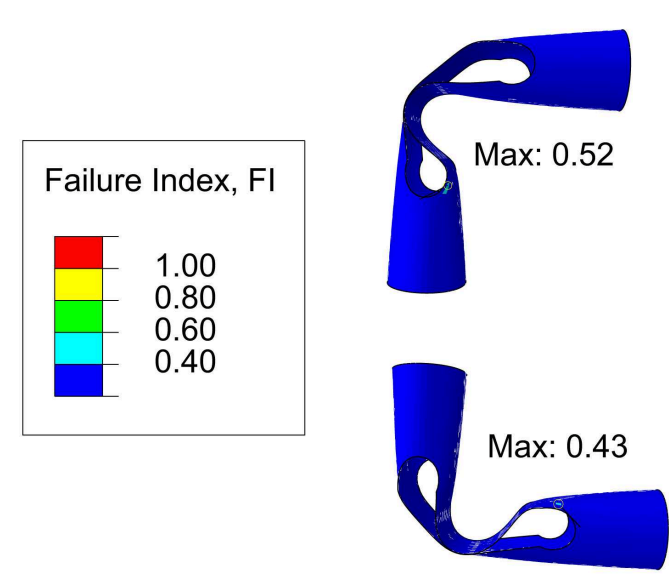

FI-1

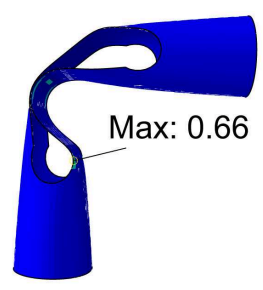

Max: 0.44

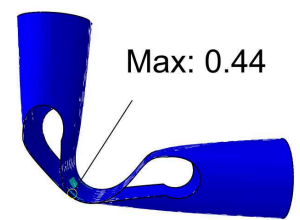

FI-2
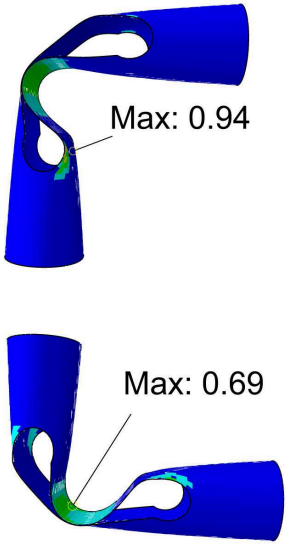

$\mathrm{FI}-3$

Figure 12: Failure indices for root hinge (top) and second hinge (bottom) in fully folded configuration, for hinge design I.
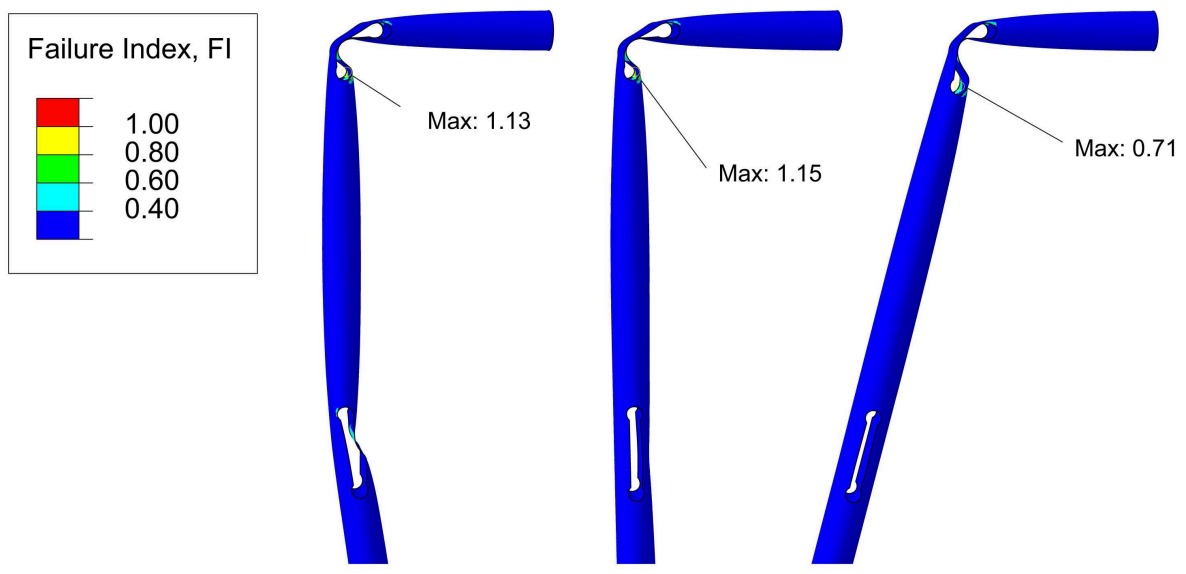

Figure 13: Maximum failure index at three stages of deployment, for boom based on hinge design I.

\begin{tabular}{|c|c|c|}
\hline Design & Fully folded & During deployment \\
\hline I & 0.94 & 1.15 \\
II & 0.81 & 1.05 \\
III & 0.92 & 0.95 \\
\hline
\end{tabular}

Table 3: Values of maximum failure index (FI-3) for three hinge designs. 


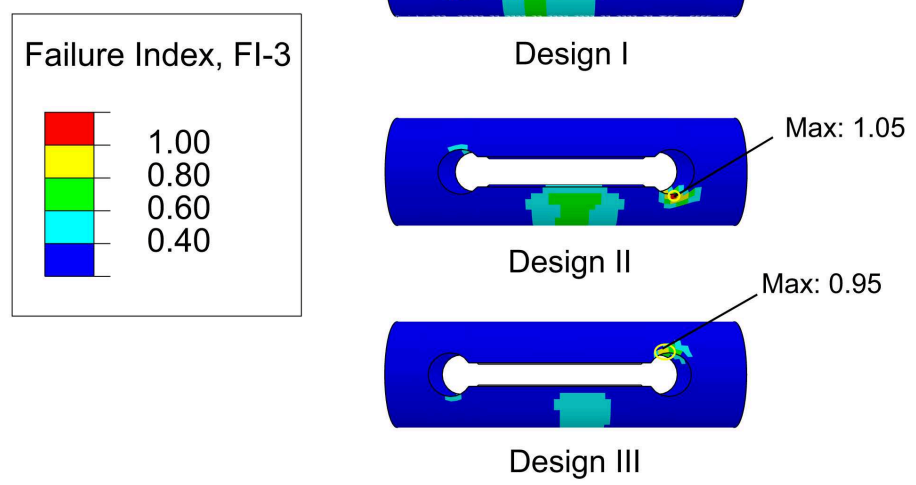

Figure 14: Location of maximum failure indices during deployment corresponding to Table 3, plotted on undeformed configuration.

Figure 15 compares the hinge angle vs. time responses of the three boom designs. Note that design I overshoots the fully deployed configuration, whereas both design II and design III latch straight away. Considering the results of the failure analysis and also the hinge angle vs. time response, design III was selected as the final design of the boom.

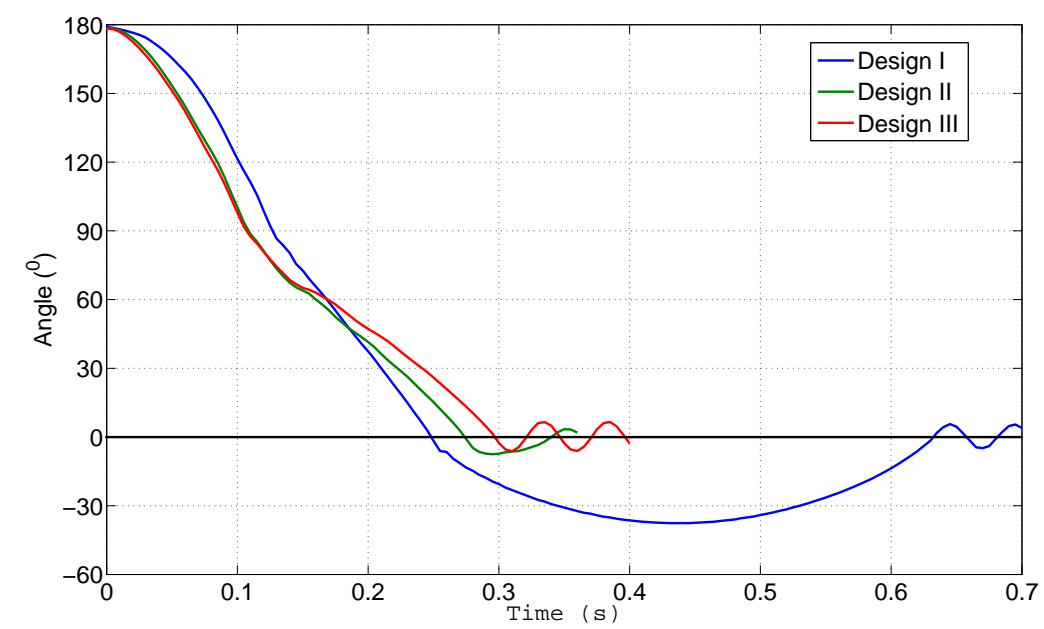

Figure 15: Comparison of hinge angle vs. time responses of the second hinge.

\section{Experimental Verification of Design III}

A boom based on Design III was built and tested to verify the accuracy of the simulation results and failure analysis on which this design had been based.

\section{A. Experimental Setup}

A $38 \mathrm{~mm}$ diameter, $1.05 \mathrm{~m}$ long and $0.22 \mathrm{~mm}$ thick boom was made from two-ply plain weave T300-1K carbon fabric impregnated with Hexply 913 epoxy resin. The fabric was laid at $45^{\circ}$ to the axis of the boom 
on a steel mandrel. The complete manufacturing procedure was described in a previous paper. ${ }^{8}$ The end $50 \mathrm{~mm}$ section of the boom was used for the root connection purposes and slots were placed as shown in Figure 16.

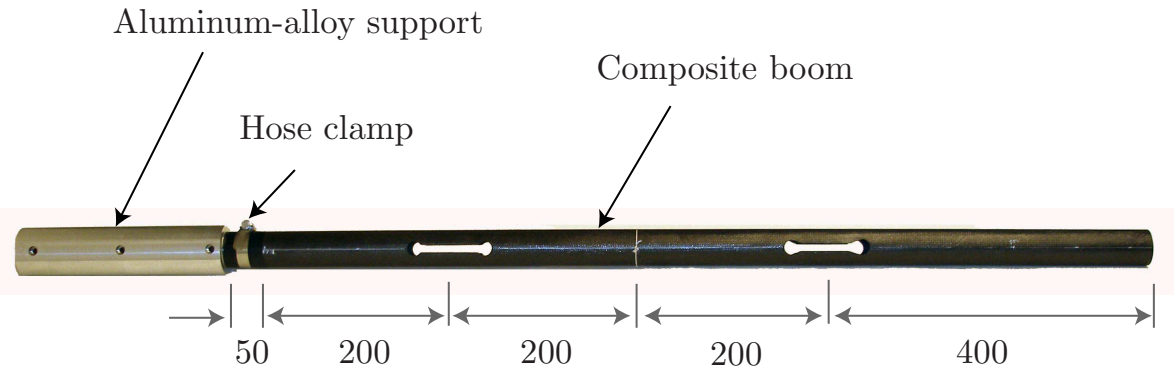

Figure 16: Complete boom (units:mm).

The root end of the boom was slid onto a solid aluminum-alloy support with a $37.8 \mathrm{~mm}$ diameter and $50 \mathrm{~mm}$ long cylindrical fitting. The overlap region was wrapped with electrical insulation tape and then clamped with a hose clamp tightened with $1.13 \mathrm{Nm}$ torque. The aluminum-alloy support was bolted to a $1 \mathrm{~m}$ by $1 \mathrm{~m}$ granite table.

This boom is able to self-deploy while carrying its own self weight however, to minimize the gravitational effects, a single-point off-load was provided through a string attached to the outer surface of the boom at a distance of $400 \mathrm{~mm}$ from the root. The other end of the string was run through a pulley located at a height of $4.65 \mathrm{~m}$ directly above the root hinge.

A $400 \mathrm{~mm}$ long, $360 \mathrm{~mm}$ wide, and $310 \mathrm{~mm}$ tall wood box was placed next to the boom to simulate the spacecraft. A Phantom V12.1 high speed camera was held directly above the root hinge with a wide angle lens to capture the detailed behavior. A Casio EX-FH20 high speed camera was placed at a height of about $4.8 \mathrm{~m}$ above the boom to capture the overall deformation. Figure 17 shows a schematic of complete experimental setup.

Great care was needed to fold the boom, specially the root hinge because the chosen design was known to have a very small margin against failure in the folded configuration. First the root hinge was pinched and folded to $90^{\circ}$ and then the same procedure was followed to fold the second hinge, Figure 18. The fully folded boom was held horizontal by hand, in the tip region, and was released while recording the deployment with the two high speed cameras, at rates of 1000 and 420 frames per second, respectively.

Snapshots of deployment were extracted from the videos recorded by the two high speed cameras and the hinge angles were measured using a specially written Matlab program that computes the angle between two lines manually drawn by an operator. This was done by loading selected pictures, defining by hand the center lines of different parts of the boom, and then computing the angle between these lines.

\section{B. Comparison}

It had been predicted by the finite element simulation that this boom should achieve its fully deployed configuration straight away, without any overshoot. Both simulation and experiment showed that the boom comes to its fully deployed configuration in about $0.3 \mathrm{~s}$, becomes fully latched, and then oscillates around the deployed configuration. The experiments also confirmed that the root hinge remains practically folded until the second hinge is completely deployed. Figure 21 shows snapshots of complete boom during the deployment experiment obtained from the second high speed camera.

Figure 20 compares the angle vs. time response of the root hinge. Note that hinge remains almost fully folded for about $0.1 \mathrm{~s}$. The second hinge deploys within this period and then remains latched throughout the rest of the experiment. The experimental and simulated angle vs. time responses show excellent agreement apart a small the initial difference, probably due to the hand release technique in the experiment. In the experiment the boom comes to its fully deployed state at $0.288 \mathrm{~s}$ whereas in the simulation it takes $0.297 \mathrm{~s}$. The final vibration portion of the response is also well predicted.

Figure 21 compares the localized deformation of the root hinge. Note that simulated response shows that after $0.105 \mathrm{~s}$ the two tape-spring hinges should swap their fold locations whereas in the experiment this 


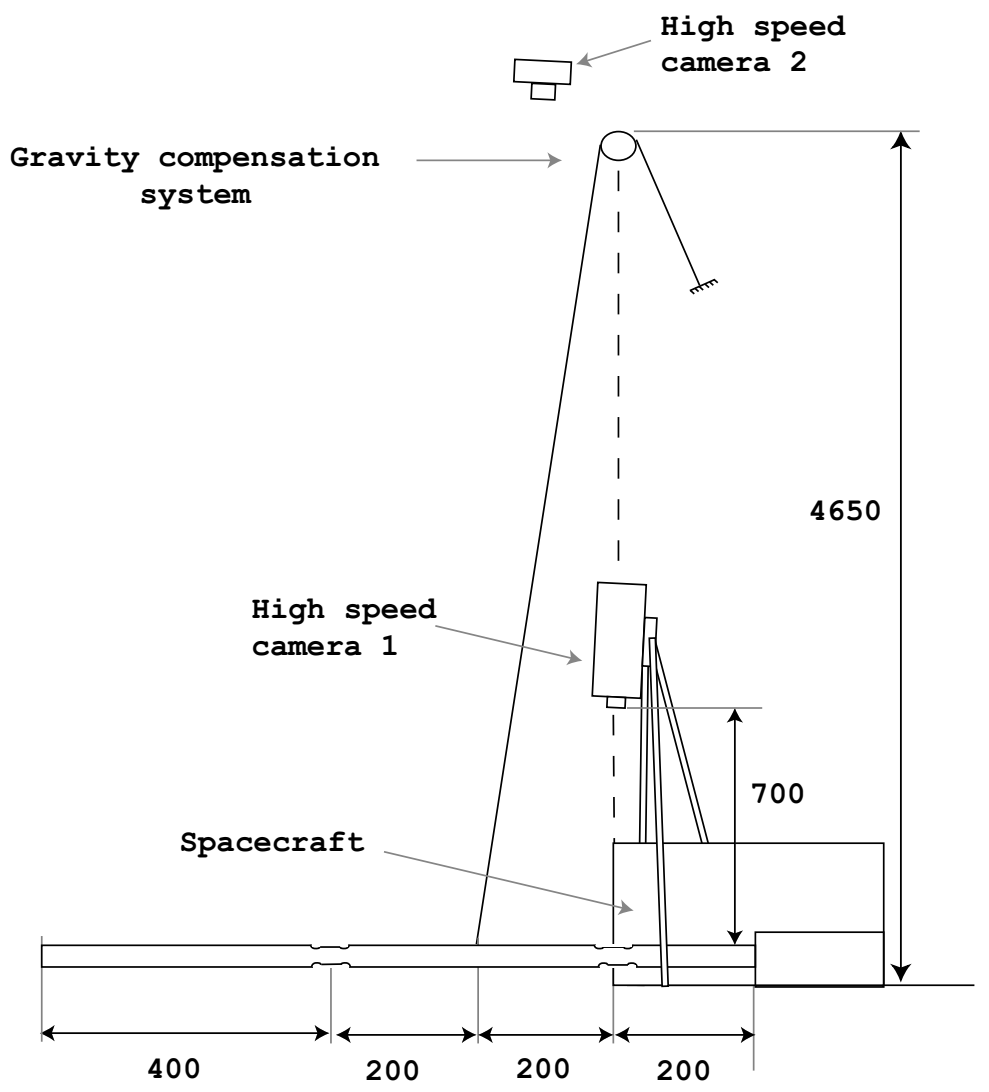

Figure 17: Experimental setup (units: mm)

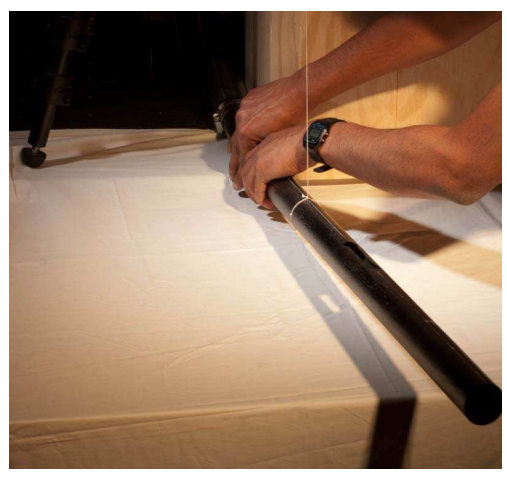

(a) Pinching root hinge

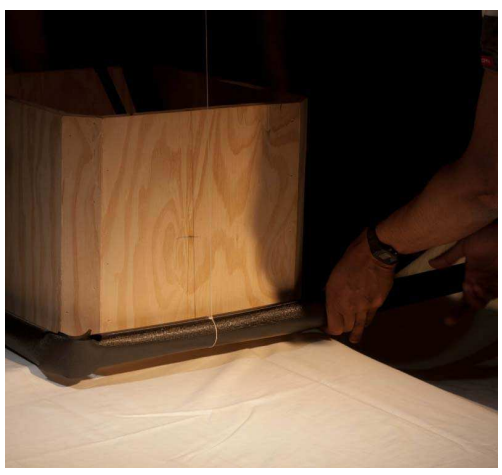

(b) Folding

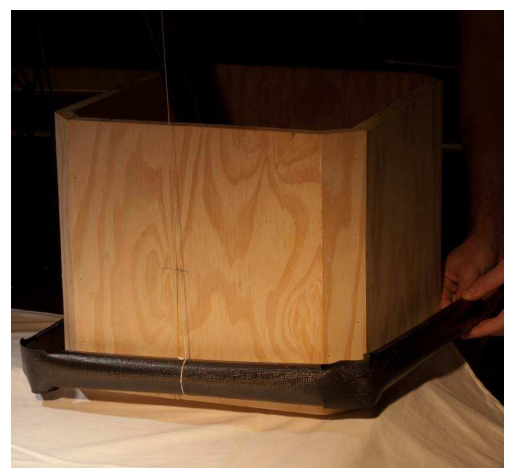

(c) Fully folded configuration

Figure 18: Setting up deployment experiment. 


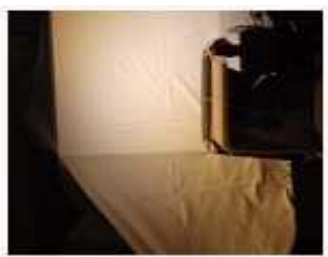

$t=0.000 \mathrm{~s}$

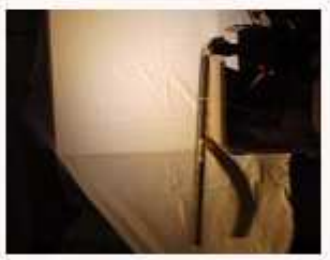

$t=0.112 \mathrm{~s}$

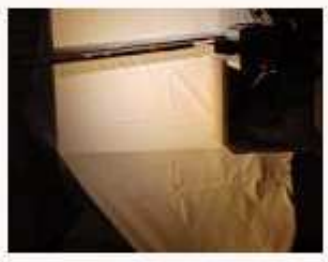

$t=0.276 \mathrm{~s}$

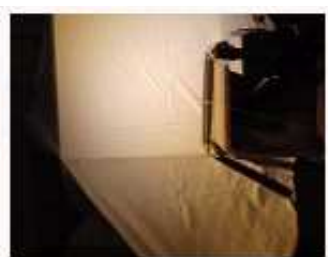

$t=0.055 \mathrm{~s}$

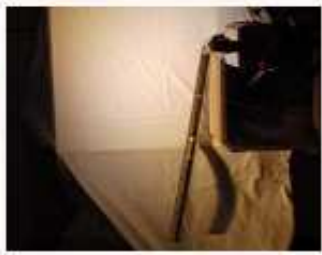

$t=0.121 \mathrm{~s}$

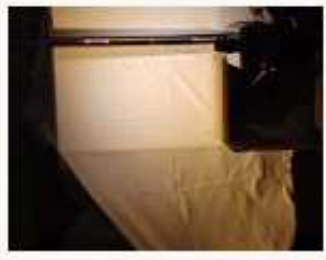

$t=0.287 \mathrm{~s}$

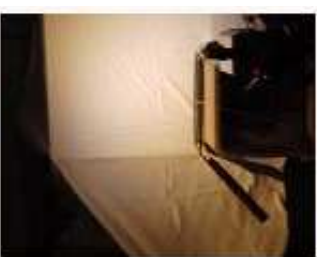

$t=0.074 \mathrm{~s}$

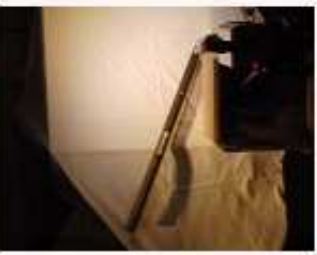

$t=0.140 \mathrm{~s}$

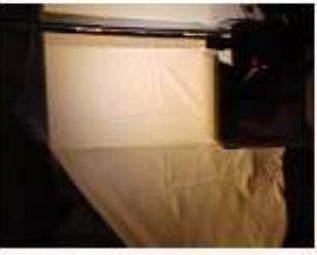

$t=0.300 \mathrm{~s}$

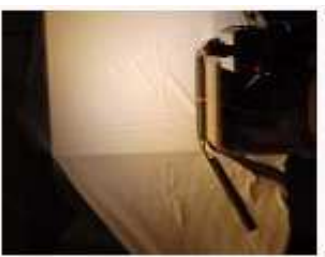

$t=0.083 \mathrm{~s}$

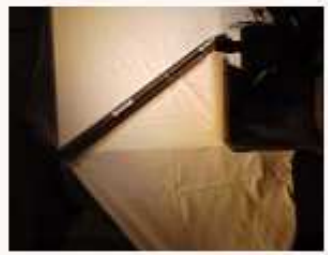

$t=0.198 \mathrm{~s}$

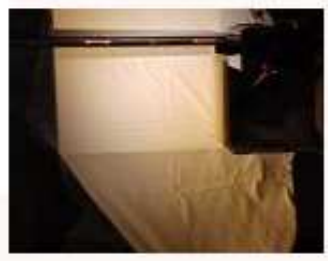

$t=0.324 \mathrm{~s}$

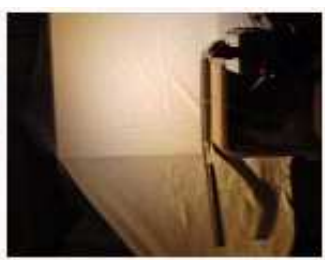

$t=0.102 \mathrm{~s}$

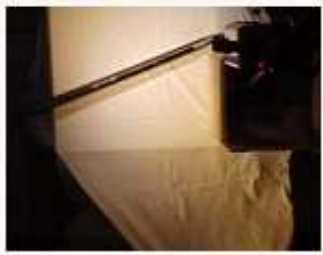

$t=0.226 \mathrm{~s}$

Figure 19: Snapshots of complete boom during deployment experiment.

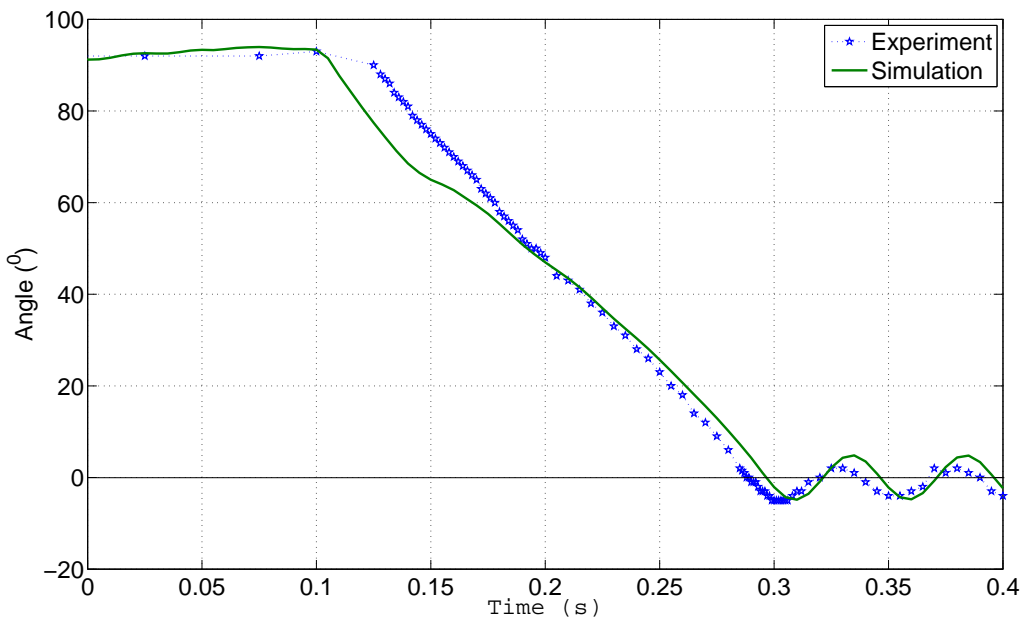

Figure 20: Comparison of root hinge angle time variation. 
change did not happen. It is known that these type of hinge has an unstable symmetric hinge configuration and hence forced to take an unsymmetric configuration. When the second hinge latches a dynamic wave travels through the boom and in the simulation this flips the root hinge configuration, however this did not happen in the experiment. The energy difference between these two alternative configurations may possibly be rather small.

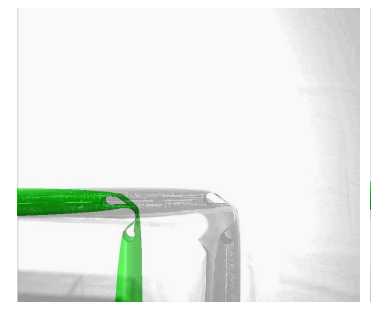

$t=0.000 \mathrm{~s}$

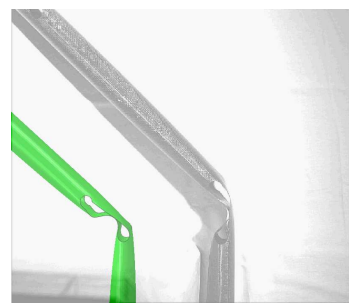

$t=0.220 \mathrm{~s}$

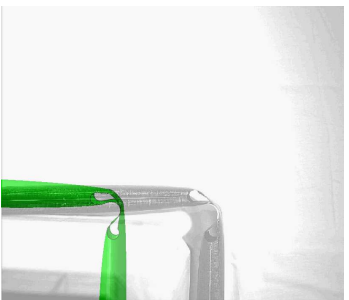

$t=0.105 \mathrm{~s}$

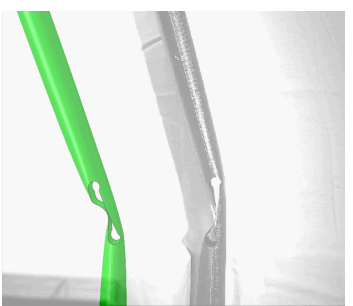

$t=0.255 \mathrm{~s}$

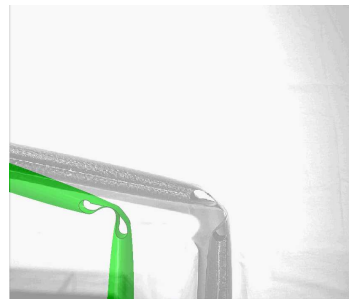

$t=0.130 \mathrm{~s}$

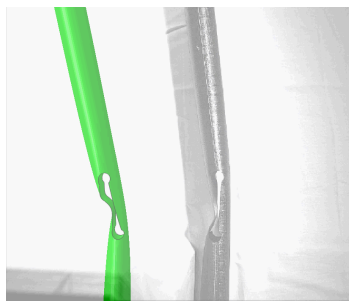

$t=0.270 \mathrm{~s}$

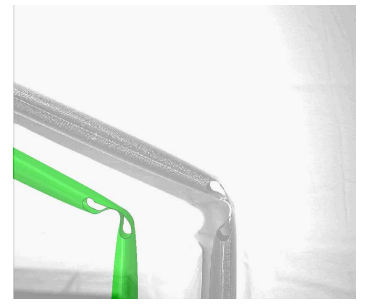

$t=0.150 \mathrm{~s}$

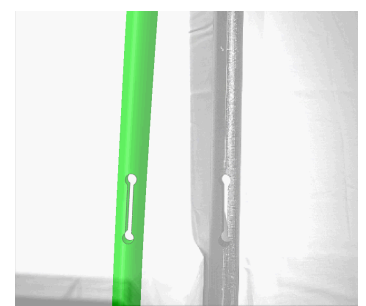

$t=0.300 \mathrm{~s}$

Figure 21: Comparison of local deformation of the root hinge.

\section{Conclusion}

This paper has utilized the simulation techniques developed in references ${ }^{7-10,17}$ to design a $1 \mathrm{~m}$ long self deployable boom that could be folded around a spacecraft. The dynamic deployment behavior of this twohinge boom, made from two-ply plain weave carbon fiber laminate, was then studied both experimentally and by means of detailed finite element simulations. A single point off-load system was used to minimize gravitational effects during the experiments.

Three possible designs of the hinges in the boom were considered, based on a previous hinge optimization study. The hinge design included a failure analysis during both stowage and dynamic deployment, using a failure criterion based on stress-resultants. This study has shown that the most critical points are the fully folded configuration and during deployment the point at which the second hinge latches, affecting the load on the root hinge. High tension and compression loads at the root hinge occur also when the root hinge latches, but we have found that hinges with two tape springs are usually strong enough to withstand this load.

The most critical portion of a hinge is near the transition region between the straight to curved part of the slot, as shown in Figure 14. These regions are subjected to both high bending and some stretching in the mid-plane and hence their strength is governed by axial-bending interaction. Therefore special care should be given to these regions during the fabrication process.

Overall we have designed and validated a $1 \mathrm{~m}$ long deployable boom with two tape-spring hinges that can be wrapped around a spacecraft and successfully deployed it without any overshoot. Our simulation techniques can be used to design deployable booms with multiple hinges and optimized boom geometry to meet any specific mission requirements. Further extensions of the present work should consider alternate laminate designs and also thermal and viscoelastic effects in the material. 


\section{Acknowledgments}

We thank Dr Michael Sutcliffe for helpful discussions and Professor Chiara Daraio and Dr Jinkyu Yang for help with high speed imaging. John Ellis (Hexcel, UK) kindly provided materials for our experiments. HM thanks the Cambridge Commonwealth Trust and the California Institute of Technology for financial support.

\section{References}

${ }^{1}$ Mobrem, M., and Adams, D.S. (2009), "Deployment Analysis of Lenticular Jointed Antennas Onboard the Mars Express Spacecraft", Journal of Spacecraft and Rockets, Vol. 46, 2009, pp. 394-402.

${ }^{2}$ Boesch, C., Pereira, C., John, R., Schmidt, T., Seifart, K., Sparr, H., Lautier, J.M., Pyttel, T. (2008) "Ultra Light SelfMotorized Mechanism for Deployment of Light Weight Space Craft Appendages," 39th Proceedings of Aerospace Mechanisms Symposium, 7-9 May 2008, Newport, NASA Marshall Space Flight Center.

${ }^{3}$ Sickinger, C., Herbeck, L., Strohelin, T., Torrez-Torres, J. (2004) "Lightweight Deployable Booms: Design, Manufacture, Verification, and Smart Materials Application," 55th International Astronautical Congress, IAF/IAA/IISL, October 04 - 08 2004, Vancouver, Canada.

${ }^{4}$ Block, J., Straubel, M. and Wiedemann, M. (2011), "Ultralight Deployable Booms for Solar Sails and Other Large Gossamer Structures in Space", Acta Astronautica, Vol. 68, 2011, pp. 984-992.

${ }^{5}$ Lane, S.A., Murphey, T.W. and Zatman, M. (2011), "Overview of the Innovative Space-Based Radar Antenna Technology Program", Journal of Spacecraft and Rockets, Vol. 48, 2011, pp. 135-145.

${ }^{6}$ Adams, D.S. and Mobrem, M. (2009) "Lenticular Jointed Antenna Deployment Anomaly and Resolution Onboad the Mars Express Spcaecraft," Journal of Spacecraft and Rockets, Vol. 46, No. 2, pp. 403-410.

${ }^{7}$ Mallikarachchi, H.M.Y.C. and Pellegrino, S. (2008) "Simulation of Quasi-Static Folding and Deployment of Ultra- Thin Composite Structures," 49th AIAA/ASME/ASCE/AHS/ASC Structures, Structural Dynamics, and Materials Conference, AIAA-2008-2053, Schaumburg, Illinois, 07-10 April 2008.

${ }^{8}$ Mallikarachchi, H.M.Y.C. and Pellegrino, S. (2009) "Deployment Dynamics of Composite Booms with Integral Slotted Hinges," 50th AIAA/ASME/ASCE/AHS/ASC Structures, Structural Dynamics, and Materials Conference, AIAA-2009-2631, Palm Springs, California, 4 - 7 May 2009.

${ }^{9}$ Mallikarachchi, H.M.Y.C. and Pellegrino, S. (2010) "Optimized Designs of Composite Booms with Tape Spring Hinges," 51st AIAA/ASME/ASCE/AHS/ASC Structures, Structural Dynamics, and Materials Conference, AIAA-2010-2750, Orlando, Florida, 12 - 15 April 2010.

${ }^{10}$ Mallikarachchi, H.M.Y.C. and Pellegrino, S. (2011) "Quasi- Static Folding and Deployment of Ultra-Thin Composite Tape-Spring Hinges," Journal of Spacecraft and Rockets, Vol. 48, 2011, 187-198.

${ }^{11}$ Soykasap, O. (2006), "Micromechanical models for bending behaviour of woven composites", Journal of Spacecraft and Rockets, Vol. 43, 2006, 1093-1100.

${ }^{12}$ SIMULIA, ABAQUS/Explicit Version 6.9, Providence, RI.

${ }^{13}$ Belytschko, T., Liu, W.K. and Moran, B. (2000) "6.2.3 Energy balance," Nonlinear Finite Elements for Continua and Structures, Chichester, J. Wiley \& Sons., pp. 315-316.

${ }^{14}$ Yee, J.C.H. and Pellegrino, S., "Folding of woven composite structures," Composites Part A-Applied Science and Manufacturing, Vol. 36, No. 2, 2005, pp. 273-278.

${ }^{15}$ Sanford, G., Biskner, A. and Murphey, T.W. (2010) "Large Strain Behavior of Thin Unidirectional Composite Flexures," 51st AIAA/ASME/ASCE/AHS/ASC Structures, Structural Dynamics, and Materials Conference, AIAA-2010-2698, Orlando, Florida, 12 - 15 April 2010.

${ }^{16}$ Tsai, S.W. and Wu, E. M.(1971), "A General Theory of Strength for Anisotropic Materials", Journal of Composite Materials, Vol. 5, 1971, 58-80.

${ }^{17}$ Mallikarachchi, H.M.Y.C. and Pellegrino, S., "Failure Crterion for Symmetric Two-Ply Plain Weave CFRP Laminates" in preperation 